\title{
Aprendizaje interprofesional en los internados de las carreras de la salud del Hospital Josefina Martínez
}

\author{
Interprofessional learning of interns at Josefina Martínez Hospital
}

\author{
Patricio Barañao ${ }^{1,2}$, Ignacio Villagrán², José Moya², Paz Jeldes², Fernanda Calvo², Eduardo Fuentes-López² , Salesa Barja1,3
}

\section{Resumen}

Introducción: las dinámicas de atención en salud requieren que los profesionales que componen el equipo de salud trabajen en forma colaborativa. Sin embargo, durante su formación, las oportunidades de aprendizaje con otras disciplinas son escasas. Se reportan los resultados del primer proyecto para promover el aprendizaje interprofesional en los internos que rotan por el Hospital Josefina Martínez. Método: de marzo a julio 2018, se realizó un taller constituido por dos sesiones, en cada rotación de internado. Participaron internos de cinco carreras en forma conjunta (kinesiología, odontología, nutrición, fonoaudiología y terapia ocupacional). Se adaptó la encuesta de disposición al aprendizaje interprofesional, para ser aplicada antes y después de cada taller. Se calcularon las medianas, rangos intercuartílicos (Q1; Q3 y se compararon los puntajes mediante test de Wilcoxon. Resultados: hubo una disposición positiva inicial al aprendizaje interprofesional, con mejoría significativa después de los talleres en todas las dimensiones de la encuesta: Trabajo en equipo y colaboración $(<0,001)$, trabajo centrado en el paciente $(<0,001)$, y sentido de identidad profesional $(<0,05)$. La diferencia del puntaje global de la encuesta también fue significativa $(<0,001)$. Conclusiones: se demostró un cambio favorable y significativo en las respuestas de los internos después de los talleres, en todas las dimensiones de la encuesta. En los comentarios, destacaron la relevancia de aprender con estudiantes de otras carreras, describiendo que aumentó su conocimiento sobre éstas y reforzando el impacto que dicha integración tiene en mejorar la atención y resolución de problemas en salud.

Palabras clave: docencia clínica; educación interprofesional; trabajo en equipo; internado; educación en ciencias de la salud; interdisciplina.

\begin{abstract}
Background: The dynamics of health care require that the health professionals work collaboratively; however, during their training, learning opportunities with other disciplines are infrequent. The results of the first project to promote interprofessional learning in the clinical internship at Hospital Josefina Martínez are reported. Methods: From March to July 2018, two workshops for each internship rotation were carried. Students from five careers participated together (Kinesiology, Dentistry, Nutrition, Speech Therapy, and Occupational Therapy). The readiness to interprofessional scale survey was adapted, to be applied before and after each workshop. Medians, interquartile range $(\mathrm{Q} 1 ; \mathrm{Q} 3)$ were calculated, and the Wilcoxon test was used to compare the results. Results: There was an initial positive readiness for interprofessional learning, with a significant improvement in all the dimensions of the survey, after the workshops: Teamwork and collaboration (<.001), patient-centered care $(<.001)$, and sense of professional identity $(<.05)$. The differences from the overall survey were also significant $(<0.001)$. Conclusions: A favorable change was demonstrated in the responses of the students after the workshops, overall and in all the dimensions of the survey. In the comments, the students highlighted the relevance of learning with students from other careers, describing that it increased their knowledge about different professions and strengthening the impact that this integration has in improving attention and resolving health problems.
\end{abstract}

Keywords: clinical teaching; interprofessional education; teamwork, clinical internship; health science education; interdiscipline.

Fecha de aceptación: 2020-05-19 - Fecha de envío: 2020-11-12

(1) Hospital Josefina Martínez.

(2) Departamento Ciencias de la Salud, Facultad de Medicina, Pontificia Universidad Católica de Chile.

(3) Departamento de Gastroenterología y Nutrición Pediátrica, División de Pediatría. Facultad de Medicina,

Pontificia Universidad Católica de Chile, Santiago de Chile.

Autor de correspondencia: invillagran@uc.cl 


\section{Introducción}

La complejidad de la atención en salud requiere actualmente que los distintos profesionales que componen el equipo de salud trabajen en forma colaborativa para lograr el máximo beneficio para los pacientes (Reeves, 2016). Sin embargo, durante el proceso formativo, las oportunidades de aprendizaje en conjunto con otras disciplinas son escasas, aun cuando la práctica profesional de atención en salud requiere del trabajo interprofesional para una mejor evaluación y tratamiento (Orchard \& Bainbridge, 2016). Esta premisa es apoyada por el Comité de Calidad en Atención en Salud del Instituto Norteamericano de Medicina (2003), el cual establece que "los equipos de salud que trabajan en forma interprofesional saben comunicarse mejor y resolver las necesidades complejas y desafiantes del contexto laboral" (Institute of Medicine (US) Committee on the Health Professions Education Summit, 2003).

La Organización Mundial de la Salud en 2010 definió que"la práctica interprofesional ocurre cuando múltiples trabajadores de la salud con diferentes antecedentes profesionales trabajan en conjunto con pacientes, familias, cuidadores y comunidades para entregar una atención de alta calidad"(World Health Organization, 2010). Para lograr equipos de trabajo interprofesional, se requiere que esta colaboración comience tempranamente en el pregrado, enfoque conocido como Educación Interprofesional (EIP) (Bridges et al., 2011). La EIP ocurre cuando estudiantes de dos o más profesiones aprenden sobre, desde y con los demás (Gilbert et al.,2010) y al implementarse, mejora la práctica futura y el cuidado de los pacientes (Abu-Rish et al., 2012).

La Real Academia Española define el concepto"interprofesional" como: "que afecta o se refiere a varias profesiones en su relación mutua", mientras que "interdisciplinario" corresponde a un "estudio o actividad que se realiza con la cooperación de varias disciplinas (ciencias o artes)". Sin embargo, ambos términos han sido utilizados para describir la colaboración entre profesionales de la salud (Mahler et al., 2014).

En Chile muchas universidades imparten las carreras del área de la salud, contexto en el cual los estudiantes comparten espacios y campos clínicos durante su proceso formativo y práctica profesional. Esto constituye una gran oportunidad para la EIP, que busca crear ocasiones donde dos o más profesionales aprendan con, de y acerca de cada uno (Zwafenstein et al., 1999). Recientemente, diversas experiencias internacionales han reportado beneficios del aprendizaje colaborativo en cuanto a habilidades, conocimientos y actitudes de estudiantes de ciencias de la salud (Guraya \& Barr, 2018; Kangas et al., 2018), pero no disponemos de información en nuestro país.
El trabajo interprofesional que se desarrolla en el Hospital Josefina Martínez (HJM), busca lograr la rehabilitación integral y transición al hogar de niños con enfermedades respiratorias crónicas complejas, en su mayoría con vía aérea artificial (Vega-briceño et al., 2004). Estos pacientes son parte de los llamados "niños y adolescentes con necesidades especiales de atención en salud", o NANEAS, (Flores et al., 2016) y proceden de unidades de cuidado intensivo y neonatología de los hospitales del sistema público de salud de Chile. En este campo clínico asistencial-docente de la facultad de medicina de la Pontificia Universidad Católica de Chile (PUC), realizan rotaciones los internos de diferentes carreras de la Salud.

Considerando que la EIP ayuda a formar profesionales con mejores habilidades para trabajar en equipo (Reeves, 2016) y que esta visión debe estar presente desde la educación de pregrado, el propósito de este estudio fue evaluar en el HJM la disposición al aprendizaje interprofesional de los estudiantes de 5to año de carreras de la Salud y su cambio después de la implementación de un taller basado en educación interprofesional.

\section{Metodología}

Estudio prospectivo e intervencional que constó de tres fases:

En la fase 1 un grupo interprofesional conformado por académicos del HJM y del departamento de Ciencias de la Salud PUC, planificó un taller de aprendizaje interprofesional definiendo objetivos, metodologías a utilizar y recursos. Para cumplir los objetivos, y debido a que los internos asisten al campo clínico durante 8 a 10 semanas, el equipo docente definió desarrollar el taller en dos sesiones por cada rotación. A continuación, se adaptó la encuesta de disposición al aprendizaje interprofesional (RIPLS, readiness interprofessional learning scale) (Reid et al., 2006), autorizado su uso por los autores, para ser aplicada antes y después de cada taller. Al inicio de este trabajo se realizó un proceso de validación de la encuesta al español chileno, que se encuentra en etapa de envío a publicación. La encuesta consta de 24 ítems y 3 dimensiones con escala Likert de 1 a 5 (en material anexo), incorporándose además 2 preguntas abiertas.

En la fase 2, se realizó un taller piloto de dos sesiones, que permitió identificar problemas e incorporar sugerencias de los estudiantes y tutores, definiendo la estructura final del taller (Tabla 1). Finalmente, en la fase 3 se realizó el taller, desarrollado en 2 sesiones, en forma sistemática a lo largo del año curricular de 2018 
Barañao et al.

Tabla 1: Estructura de los talleres de aprendizaje interprofesional.

\begin{tabular}{|c|c|c|}
\hline Sesión & Objetivo & Metodología \\
\hline 1 & $\begin{array}{l}\text { Describir los roles de los diferentes profe- } \\
\text { sionales del equipo de salud. }\end{array}$ & $\begin{array}{l}\text { - Presentación invertida: Los estudiantes se presentaban en pares de distinta carrera en } \\
\text { base a preguntas preestablecidas, y luego debían presentar al par frente al grupo (1) } \\
\text { - Historia de mi profesión en el mundo y en Chile, con presentación de una infografía } \\
\text { con línea de tiempo, por grupo de pares de igual carrera. }\end{array}$ \\
\hline 2 & $\begin{array}{l}\text { Aplicar aspectos del profesionalismo en el } \\
\text { trabajo interprofesional }\end{array}$ & $\begin{array}{l}\text { Definir nueve valores a centrales del profesionalismo a partir de bibliografía en- } \\
\text { tregada y búsqueda personal (trabajo previo). } \\
\text { Reconocimiento de valores del profesionalismo: Dramatización con ejemplos de } \\
\text { una situación en un hospital o en atención ambulatoria. } \\
\text { - Análisis de videos con ejemplos de trabajo interprofesional: destacar fortalezas y } \\
\text { debilidades de situaciones contrapuestas. }\end{array}$ \\
\hline
\end{tabular}

a Los nueve valores centrales del profesionalismo desarrollados fueron: 1) Altruismo, 2) Responsabilidad, 3) Excelencia, 4) Obligación y apoyo, 5) Servicio, 6) Honor, 7) Integridad, 8) Respeto por los demás y 9) Estándares éticos y morales.

\section{Participantes e implementación}

Tanto el taller piloto como los cuatro talleres posteriores se realizaron en el HJM, desde marzo a octubre de 2018, correspondiendo a un taller por cada rotación de internado. Cada taller constó de 2 sesiones. La muestra fue por conveniencia y participaron en forma conjunta los internos de cinco carreras de la salud: kinesiología, odontología, nutrición, fonoaudiología y terapia ocupacional.

\section{Recolección de la información}

En cada taller se entregó la encuesta antes de la $1^{\text {a }}$ sesión y después de finalizada la $2^{2}$ sesión para ser contestada en forma presencial. La encuesta RIPLS adaptada consistió en 24 ítems con escala Likert (1 a 5, siendo 1 Muy en desacuerdo y 5 Muy de acuerdo). En la aplicada al final se incluyeron además dos preguntas abiertas: (1. ¿Cuáles fortalezas destacas del taller realizado? ¿2. Cuáles aspectos por mejorar identificas?).

\section{Análisis estadístico}

Se analizó la distribución de las variables con test de Shapiro-Wilk arrojando una distribución no normal $(p<0,05)$. Por lo tanto, se describió la distribución por medio de las medianas, y su dispersión mediante los rangos intercuartílicos (Q1; Q3). Se aplicó test de Wilcoxon para comparar los resultados pre y post intervención.
Se consideró como significativo un valor $\mathrm{p}<0,05$. El análisis se realizó con el programa SPSS v22 (IBM Corp, 2013).

\section{Resultados}

Durante el período de estudio, marzo a octubre 2018 realizaron su rotación por el hospital 64 alumnos del último año de pregrado de carreras de ciencias de la salud. De ellos, dos realizaron rotaciones en dos ocasiones por el Hospital Josefina Martínez durante el período mencionado, participando solamente en la primera de éstas.

El número total de alumnos participantes fue de 62 , realizándose en total 5 talleres de dos sesiones cada uno. Doce estudiantes participaron en el taller piloto y 50 en los 4 talleres analizados. Finalmente, 2 encuestas no se incluyeron por estar incompletas, quedando un total de 48 encuestas analizadas, al excluirse las del piloto. La distribución por género fue de 42 mujeres $(87,5 \%)$ y 6 hombres (12,5\%). La mediana (Q1;Q3) de la edad fue 23 años (22;24). El número de estudiantes por carrera se describe en la Tabla 2.

En cuanto a las tres dimensiones de la encuesta, se encontró un aumento del puntaje, con diferencia significativa en los resultados antes y después de los talleres de aprendizaje interprofesional. No hubo diferencias entre carreras ni entre los talleres entre sí (Tabla 3). 
Barañao et al.

Tabla 2: Número y carrera de 62 internos participantes en los talleres de educación interprofesional en el HJM.

\begin{tabular}{|c|c|c|c|c|c|c|}
\hline \multirow{2}{*}{ Talleres } & \multicolumn{5}{|c|}{ Carreras } & \multirow[b]{2}{*}{ Total } \\
\hline & Fonoaudiología & Kinesiología & Nutrición & Odontología & T.Ocupacional & \\
\hline $1^{*}$ & 2 & 4 & 2 & 2 & 2 & 12 \\
\hline 2 & 2 & 5 & 3 & 2 & 2 & 14 \\
\hline 3 & 2 & 5 & 3 & 0 & 0 & 10 \\
\hline 4 & 2 & 5 & 2 & 2 & 2 & 13 \\
\hline 5 & 2 & 4 & 3 & 2 & 2 & 13 \\
\hline Total (\%) & $10(16,1)$ & $23(37,1)$ & $13(21)$ & $8(12,9)$ & $8(12,9)$ & (100) \\
\hline \multicolumn{7}{|c|}{ * Taller piloto } \\
\hline \multicolumn{3}{|l|}{ Dimensión } & $\begin{array}{l}\text { Pre-taller } \\
\text { Mediana }(Q 1 ; Q 3)\end{array}$ & \multicolumn{2}{|c|}{$\begin{array}{l}\text { Post-taller } \\
\text { Mediana }(\mathrm{Q} 1 ; \mathrm{Q} 3)\end{array}$} & p-value \\
\hline \multicolumn{2}{|c|}{ Trabajo en equipo y colaboración } & \multicolumn{2}{|r|}{$4,8(4,5 ; 5,0)$} & $5,0(4,9$ & \multicolumn{2}{|r|}{$<0,001$} \\
\hline \multicolumn{2}{|c|}{ Centrado en el paciente } & \multicolumn{2}{|r|}{$4,5(4,2 ; 4,8)$} & $4,5(4,3$ & \multicolumn{2}{|r|}{$<0,001$} \\
\hline \multicolumn{2}{|c|}{ Sentido de identidad profesional } & \multicolumn{2}{|r|}{$5,0(4,4 ; 5,0)$} & $5,0(4,8$ & \multicolumn{2}{|r|}{$<0,05$} \\
\hline \multicolumn{2}{|l|}{ Total } & \multicolumn{2}{|r|}{$4,6(4,5 ; 4,8)$} & $4,8(4,7$ & \multicolumn{2}{|r|}{$<0,001$} \\
\hline
\end{tabular}

En el aspecto cualitativo de la encuesta, para la primera pregunta “Cuáles fortalezas destacas del taller de aprendizaje interprofesional?", las respuestas más frecuentes se focalizaron en que esta actividad les permitió conocer más a las otras disciplinas. Las opiniones respecto a temas de relevancia común fueron:

- "Bueno conocer e interactuar con profesionales o estudiantes de otras áreas" (E3, T3).

- "Ayuda a comprender la importancia del trabajo interprofesional" (E5, T4).

- "Conocer el rol y punto de vista de las otras carreras del equipo de salud" (E8, T2).

En las respuestas a la segunda pregunta: ¿Cuáles aspectos a mejorar identificas?), se destaca la necesidad de perfeccionar las estrategias de enseñanza del taller, sugiriendo incorporar metodologías activas de integración, análisis y aplicación de lo aprendido:

- "Hablar más de la experiencia en el internado en vez de definiciones" (E10, T5).
- $\quad$ "Generar momentos donde los estudiantes puedan explicar con profundidad su labor" (E9, T3).

- "Simular una reunión clínica interdisciplinaria como actividad final para poder aplicar lo aprendido" $(\mathrm{E} 3, \mathrm{~T} 4)$.

- Otro aspecto a mejorar descrito fue la inquietud sobre que este tipo de actividades debiera implementarse en forma previa dentro de los planes de estudio:

- "Debe realizarse desde el inicio de la carrera, más que al final" (......).

- "No se debiese hacer en esta instancia, sino que desde inicios de pregrado" (......)

\section{Discusión}

Este estudio reporta la aplicación de un taller de aprendizaje interprofesional a alumnos del último año de pregrado, de cinco carreras de la salud de la Pontificia Universidad Católica de Chile, y la evaluación de su percepción de antes y después de la actividad 
educativa. Constituye una experiencia novedosa en nuestro país, en el contexto de la complejización del cuidado en salud y su optimización mediante el trabajo interprofesional.

El trabajo en equipo inteprofesional para pacientes con necesidades complejas es un desafío para los profesionales (Vries et al., 2017), pero se requieren también otras condiciones para que éste se lleve a cabo. El Hospital Josefina Martínez reune características facilitadoras para el desarrollo de la EIP, ya que los internos de distintas profesiones de la salud realizan sus rotaciones en forma simultánea, en cercanía a los diferentes tutores clínicos. Se busca una atención integral centrada en el paciente y su familia (Flores et al., 2016), lo que favorece instancias para el desarrollo de competencias necesarias para el modelaje de la comunicación y trabajo en equipo (Interprofessional Education Collaborative, 2016). Asimismo, el HJM cuenta con áreas comunes fuera del espacio clínico, donde se realizan seminarios de temas transversales y de convivencia, lo cual se necesita para realizar actividades de EIP (Bridges et al., 2011). Finalmente, la realización de este taller ha sido apoyado por la dirección del HJM, la subdirección de docencia y los respectivos docentes tutores de internado, reconociendo que la promoción del trabajo interprofesional requiere de un compromiso permantente de las jefaturas de cada servicio, favoreciendo las instancias de colaboración interprofesional y promoviendo un lenguaje común respecto a la atención de los pacientes (Vries et al., 2017).

Respecto a la implementación del taller, la primera sesión consideró la historia en el mundo y en Chile de las profesiones participantes, para el reconocimiento inicial de los otros roles dentro del equipo de salud, puesto que los estudiantes no habían tenido oportunidad de interacción durante los años previos. La segunda sesión se enfocó en el análisis de los valores centrales del profesionalismo (Mcnair, 2005), para favorecer la coordinación en la comunicación y especialmente, el cuidado de los pacientes y sus familias (Holtman et al., 2011).

El análisis de los resultados demostró que, si bien al inicio hubo una disposición favorable de los internos al aprendizaje interprofesional, se produjo una mejoría significativa después de los talleres, global y en todas las dimensiones de la encuesta. Estos resultados coinciden con lo reportados por Guyara con estas metodologías (Guraya \& Barr, 2018). La dimensión de "trabajo en equipo y colaboración" tuvo el mayor incremento, lo cual podría explicarse por la naturaleza colaborativa del Hospital Josefina Martínez y por la participación de internos de 5 carreras de la salud asociado a una dinámica muy participativa incorporada en el taller. Por el contrario, la dimensión "sentido de identidad profesional" fue la que tuvo menor aumento, debido posiblemente a que la percepción inicial de los estudiantes en este ámbito ya era superior. También puede influir el que los participantes correspondían a estudiantes de último año de cada carrera, por lo tanto, se han enfrentado previamente a experiencias clínicas donde se observa el aporte de cada disciplina al bien común y se podría haber desarrollado una identidad del propio rol. Dicho factor ha sido reportado como el de resultados más variables entre los autores que utilizan RIPLS (El-Zubeir et al., 2006).

En el aspecto cualitativo, los estudiantes destacaron la relevancia de aprender con pares de otras carreras durante el internado, describiendo que aumentó su conocimiento sobre éstas, y reforzando el impacto que esta integración tiene en mejorar la atención y resolución de problemas en salud. La literatura describe que la educación interprofesional ayuda a los estudiantes a clarificar los roles del equipo de salud y mejora positivamente sus actitudes hacia el trabajo interprofesional (Reeves et al., 2013; Illingworth \& Chelvanayagam, 2017), lo que podría permitirles abordar en forma más efectiva e integral las necesidades y desafíos que se verán enfrentados en el ambiente clínico-laboral (Orchard \& Bainbridge, 2016).

A nivel internacional, se han reportado numerosos problemas en la coordinación y colaboración entre los diferentes profesionales que conforman el equipo de la salud, así como su impacto sobre la calidad de la atención recibida por el paciente ( Reeves, 2016; Guraya \& Barr, 2018). Esto ha aumentado el interés y destinación de recursos hacia la educación interprofesional por los efectos positivos sobre las habilidades de trabajo colaborativo (Guraya \& Barr, 2018)

Son fortalezas del presente trabajo su originalidad y coincidencia con estas tendencias en educación en ciencias de la salud, que reconocen el trabajo interprofesional como la mejor estrategia de tratamiento. Otro aspecto beneficioso es haber reunido internos de cinco carreras de la salud, si bien destaca una mayor proporción $(36,7 \%)$ de alumnos de kinesiología. Esto se explica por la naturaleza de las patologías de los pacientes de nuestro hospital, que requieren particularmente de la rehabilitación cardiorespiratoria y neuromotora, lógica, lo cual origina una mayor cantidad de docentes e internos de kinesiología en ambas áreas. A pesar de dicha distribución, la variabilidad de la respuestas fue baja entre las distintas carreras y no representó un factor relevante al momento de analizar los resultados.

Una limitación de nuestro estudio es el no haber contado con la participación de internos de las carreras de medicina y enfermería, puesto que hasta la fecha no existe rotación de los primeros en HJM, y que no hubo rotación en dicho período de internos de enfermería. 
Por otro lado, es necesario considerar que si utilizamos la tipología expandida de Kirkpatrick para la evaluación de intervenciones de educación interprofesional (Reeves et al., 2015), nuestro trabajo sólo logra evaluar los niveles 1 (Percepción de los estudiantes) y 2A (modificación de actitudes/percepciones). Sin embargo, al ser una experiencia preliminar, estos resultados pueden sentar las bases para planificar estrategias que busquen evaluar niveles de Kirkpatrick más altos, como son la adquisición de conocimientos y habilidades, cambios en la práctica organizacional y finalmente, los beneficios que la EIP podría tener en los pacientes, sus familias y comunidades. Con este trabajo, esperamos colaborar y motivar la incorporación de la EIP en todos los programas de pregrado de la facultad de medicina de la PUC, en forma sistematizada y coherente con el plan de estudios, desde el primer al último año de formación académica para todas las carreras de la salud.

\section{Conclusión}

Este proyecto logró convocar a un grupo significativo de estudiantes de último año de las carreras de la salud que realizaron su internado en el HJM entre marzo y octubre de 2018. Se demostró una mejoría en la disposición al aprendizaje interprofesional después de participar en el taller de aprendizaje interprofesional. Los estudiantes destacaron los talleres como primera instancia formal de aprendizaje con estudiantes de otras carreras durante el pregrado, y el posible impacto de esta integración para mejorar la atención y resolución de problemas en salud.

\section{Fuentes de financiamiento}

Este proyecto fue financiado por:

- Fundación Josefina Martínez de Ferrari.

- FONDEDOC 2019-2020 Ignacio Villagrán “Diseño, implementación e impacto de la educación interprofesional en las carreras de Ciencias de la Salud".

\section{Agradecimientos}

A todos los docentes tutores de internado del hospital Josefína Martínez, por compatibilizar los horarios del internado con los del taller.

A la secretaria del modulo docente, por su constante apoyo a los internos y en la logística para desarrollar la actividad.

\section{Contribuciones y conflictos declarados por los autores}

Todos los autores declaran no tener conflicto de interés en este proyecto.

\section{Aspectos éticos}

El proyecto fue aprobado por el comité de ética de la facultad de medicina de la Pontificia Universidad Católica de Chile ( $n^{\circ} 181109003$ ) y los alumnos firmaron un documento de consentimiento informado previo a cada taller de aprendizaje interprofesional.

\section{Referencias}

Abu-Rish E, Kim S, Choe L, Varpio L, Malik E, White A, Craddick K, Blondon K, Robins L, Nagasawa P, Thigpen A, Chen L, Rich J. \& Zierler B. (2012). Current trends in interprofessional education of health sciences students: A literature review. Journal of Interprofessional Care 26, 444-451.

Bridges D, Davidson R, Odegard P, Maki I. \& Tomkowiak J. (2011). Interprofessional collaboration: Three best practice models of interprofessional education. Medical Education Online, 16.

El-Zubeir M, Rizk D \& Al-Khalil R. (2006). Are senior UAE medical and nursing students ready for interprofessional learning ? Validating the RIPL scale in a Middle Eastern context. Journal of Interprofessional Care 20, 619-632.

Flores J, Lizama M, Rodríguez N, Ávalos M, Galant M, Barja S, Becerra C, Sanhueza C, Cabezas A, Orellana, JorgeZillmann G, Antilef R, Cox A, Valle $M$, Vargas N \& Comité NANEAS Sociedad Chilena de Pediatría. (2016). Modelo de atención y clasificación de « Niños y adolescentes con necesidades especiales de atención en salud-NANEAS » : recomendaciones del Comité NANEAS de la Sociedad Chilena de Pediatría Revista Chilena de Pediatría 87, 224-232.

Gilbert J, Yan J. \& Hoffman S. (2010). A WHO report: Framework for action on interprofessional education and collaborative practice. Journal of Allied Health 39, 196-197.

Guraya S. \& Barr H. (2018). The effectiveness of interprofessional education in healthcare : A systematic review and meta-analysis. Kaohsiung Journal of Medical Sciences 34, 160-165.

Holtman MC, Frost JS, Hammer DP, Mcguinn K. \& Nunez LM. (2011). Interprofessional professionalism : Linking professionalism and interprofessional care. Journal of Interprofessional Care 25, 383-385.

IBM Corp. (2013). IBM SPSS Statistics for Windows, Version 22.0. Armonk, NY: IBM Corp.

Illingworth P. \& Chelvanayagam S. (2017). The benefits of interprofessional education 10 years on. British Journal of Nursing $26,813-818$. 
Institute of Medicine (US) Committee on the Health Professions Education Summit. (2003). The Core Competencies Needed for Health Care Professionals. In A. Greiner \& E. Knebel (Eds.), Health Professions Education: A Bridge to Quality. Washington (DC): National Academies Press.

Interprofessional Education Collaborative. (2016). Core Competencies for Interprofessional Collaborative Practice: 2016 update. Washington, DC: Interprofessional Education Collaborative.

Kangas S, Rintala T. \& Jaatinen P. (2018). An integrative systematic review of interprofessional education on diabetes. Journal of Interprofessional Care 24, 1-13.

Mahler C, Karstens S, Joos S. \& Gutmann T. (2014). Terminology for interprofessional collaboration: Definition and current practice. GMS Zeitschrift Fur Medizinische Ausbildung 31, 1-10.

Mcnair R. (2005). The case for educating health care students in professionalism as the core content of interprofessional education. Medical Education 39, 456-464.

Orchard C. \& Bainbridge L. (2016). Competent for collaborative practice :What does a collaborative practitioner look like and how does the practice context influence interprofessional education ?. Journal of Taibah University Medical Sciences 11, 526-532.

Reeves S, Boet S, Zierler B \& Kitto S. (2015). Interprofessional Education and Practice Guide No. 3: Evaluating interprofessional education Journal of Interprofessional Care 305-12.
Reeves S, Perrier L, Goldman J, Freeth D \& Zwarenstein M. (2013). Interprofessional education: e ects on professional practice and healthcare outcomes (Review). The Cochrane database of systematic reviews CD002213.

Reeves S. (2016). Why we need interprofessional education to improve the delivery of safe and effective care. Interface (Botucatu) 20, 185-196.

Reid R, Bruce D, Allstaff K. \& McLernon D. (2006). Validating the Readiness for Interprofessional Learning Scale (RIPLS) in the postgraduate context: are health care professionals ready for IPL?. Medical Education 40, 415-422.

Vega-briceño L, Contreras I, Prado F, Méndez M. \& Sánchez I. (2004). Hospital Josefina Martínez : Una breve reseña histórica. Neumología pediátrica 1, 3-5.

Vries A, Jean J. \& Van Dongen J. (2017). Sustainable interprofessional teamwork needs a team-friendly healthcare system : Experiences from a collaborative Dutch programme. Journal of Interprofessional Care 31, 167-169.

World Health Organization. (2010). Framework for action on interprofessional education and collaborative practice. Accedido en: https://apps.who.int/iris/handle/10665/70185 el 19 de abril de 2020

Zwafenstein M, Atkins J, Barr H, Hammick M, Koppel I. \& Reeves S. (1999). A systematic review of interprofessional education. Journal of Interprofessional Care 13, 417-424. 